\section{Chernobyl, eight years on}

\section{Dillwyn Williams}

THE explosion at the Chernobyl nuclear reactor was an unprecedented disaster that has put the health of millions of people at risk. This is one version of events. The opposite view has it that Chernobyl was indeed a serious accident, but that although a lot of radiation was released it was quickly diluted and will have little discernible effect on the health of the exposed population. The truth, of course, is likely to lie somewhere between these two extremes.

Eight years after the accident, how do things stand? Meetings held in the past few months, and papers published, support the view that there has been a considerable rise in the incidence of thyroid carcinoma in children in the area around Chernobyl, but that there has not been a great increase in the reported incidence of other tumours or of genetic defects. Radiation is a mutagen and, like other mutagens, may exert its therapeutic effect on tumours in part through deficient repair of tumour-cell DNA. As a mutagen it has been associated with an increased risk of a wide variety of malignancies ranging from brain tumours to skin tumours and sarcomas. Two of the best documented radiation-induced malignancies are leukaemia and thyroid carcinoma.

To consider leukaemia first. Exposure to radiation is a known cause of the disease, and children are especially sensitive. No increase has been found in the incidence of childhood leukaemia in the areas around Chernobyl ${ }^{1}$ so it is reassuring but hardly surprising that no increase has been found in the Scandinavian countries $^{2,3}$ or in broader European studies ${ }^{4}$. That does not prove that no increase will occur (and the studies so far have not had the power to detect a small increase) but it makes it unlikely that there will be any major effect. A report suggesting that an isolated increase in births with trisomy 21 in Berlin was related to exposure to fallout ${ }^{5}$ differs from larger European studies ${ }^{6}$, and we do not know in how many other European cities an enterprising geneticist or paediatrician has scanned the records of births with severe genetic defects and failed to find an association with fallout from Chernobyl. If no effect is shown in an area of high exposure it is hardly likely that any effect will be shown in areas of low exposure, with a few exceptions where the radiation dose is so high that it sterilizes cells rather than causing them to become neoplastic.

In contrast to the studies of leukaemia or genetic defects, analyses of the incidence of thyroid cancer in children have been conducted mainly in the areas around Chernobyl. The first announce- ments of an increase in incidence ${ }^{7,8}$ have been followed by further documentation of the pathology 9,10 . The numbers reported have continued to increase and the tumours have been reported in northern Ukraine as well as in southern Belarus, and more recently in that part of the Russian Federation close to Chernobyl. These are a coherent group of results, in that the highest incidence and shortest latent period appears to be in the area exposed to the highest level of fallout. But they are not based on unbiased epidemiological studies, and the contribution of screening for thyroid tumours to the reported incidence is not known.

The strength of the association between the incidence of childhood thyroid cancer and exposure to fallout from Chernobyl was underlined this summer at two meetings* in Nagasaki. Continuing increases in the numbers of childhood thyroid cancer diagnosed were reported, bringing the total in Belarus, since Chernobyl, to 251 and in the Ukraine to 276. Most of the Belarussian cases have occurred over the past four years, in the Gomel region, closest to Chernobyl, where the rate of childhood thyroid cancer reported has reached over 100 per million children per year. In most national tumour registries the childhood thyroid cancer rate is between 0.5 and 3 , so the scale of the reported increase is dramatic.

The one problem preventing unqualified acceptance of the reported figures is that of ascertainment. Small papillary carcinomas, often only a millimetre or two across, are common incidental autopsy findings in adults. However, the pathology of the tumours reported from the countries involved is not that of microscopic lesions but of aggressive cancers, which are almost all papillary in type and have often directly invaded tissues around the thyroid. At one of the meetings, the result of a straw poll of an 11-strong panel of international experts was unanimity that the diagnosis of the tumours had been adequately substantiated and that exposure to the Chernobyl accident was definitely or probably the cause of the increase. Ten of the 11 considered that increased surveillance was of medium or low importance in contributing to the size of the increase.

The most likely cause of this increase in childhood thyroid cancer, with no apparent major increase in leukaemia or other tumours, is the radioiodine content of the fallout. This contrasts with the safety of ${ }^{131}$ I treatment of adults with Graves dis-

*Chernobyl Update and Chernobyl in the Future, Nagasaki, Japan. 3-4 June 1994. Proceedings to be published as Excerpta Medica International Congress Series 1074. ease. The limited growth potential of the normal follicular cell ${ }^{11}$ may well be relevant to this discrepancy. The presence in fallout close to Chernobyl of short-lived isotopes of iodine as well as ${ }^{131} \mathrm{I}$ may also be important; the amount of ${ }^{133}$ I released was almost a third of that of ${ }^{131} \mathrm{I}$. Evidence of the link is likely to be indirect; the dose to the thyroid was measured after the event in only a small proportion of those exposed, and reconstruction is complex.

Molecular epidemiology may help. The sequence of events in thyroid carcinogenesis is partially known, and for papillary carcinoma ret or trk translocations, met overexpression and ras point mutations are among the commoner findings. Radiation is more likely to cause deletions or translocations than most chemical mutagens, and a limited study ${ }^{12}$ has looked for ret translocations in childhood thyroid cancer in the Chernobyl area. The small number of cases successfully studied and lack of information on the frequency of ret translocation in childhood papillary carcinoma makes it difficult to draw conclusions. A different pattern of mutations in radiation-induced as opposed to nonradiation-induced experimental thyroid tumours has been reported ${ }^{13}$. Clearly, it is of considerable interest to compare the pattern of oncogene involvement in childhood thyroid carcinomas exposed to fallout from Chernobyl with a non-exposed control series.

Finally, study of the consequences of Chernobyl should be put into perspective. The increase in childhood thyroid cancer is relatively large, but it is not yet a serious problem in overall public health terms. Nonetheless, evidence from those exposed to external radiation or to radiation during the nuclear tests on the Marshall Islands shows that cases of thyroid cancer can occur for decades after exposure. Moreover there is a chance that the combination of human error and ageing reactors will cause a similar disaster. Analysis of the consequences of Chernobyl is essential to inform the response to any comparable event in the future.

Dillwyn Williams is in the Department of Histopathology, Addenbrooke's Hospital, Cambridge CB2 2QQ, UK.

\footnotetext{
1. Ivanov, E. P., Tolochko, G., Lazarev, V. S. \& Shuvaeva, L. Nature 365, 702 (1993)

Auvinen, A. etal. Br. Med. J. 309, 151-154 (1994).

3. Hjalmars, U. et al. Br. Med. J. 309, 154-157 (1994)

4. Parkin, D. M. et al. Eur. J. Cancer 29A, 87-95 (1993)

5. Sperling, K. et al. Br. Med. J. 309, 158-161 (1994).

6. Little, J. Paediat. perinat. Epidem. 7, 121-151(1993).

7. Kazakov, V. S., Demidchik, E. P. \& Astakhova, L. N. Nature 359, 21 (1992).

8. Baverstock, K Egloff, B., Pinchera, A., Ruchti, C. \& Williams, D. Nature 359, 21-22 (1992)

9. Furmanchuk, A. W. et al. Histopathology 21, 401-408 (1992).

10. Nikiforov, Y. \& Gnepp, D. R. Cancer 74, 748-766 (1994).

11. Wynford-Thomas, D., Stringer, B. M. J., Harach, H. R. \& Williams, E. D. Experientia 39, 421-423 (1983).

12. Ito. T. et al. Lancet 344, 259 (1994)

13. Lemoine, N. R., Mayall, E. S., Williams, E. D., Thurston, V. \& Wynford-Thomas, D. Oncogene 3, 541-544 (1988)
} 